\title{
Go West or Rest! Die Behandlung chronischer Erschöpfungssyndrome im Wandel der Zeiten
}

\author{
Jens Gaab \\ Klinische Psychologie und Psychotherapie, Fakultät für Psychologie, Universität Basel, Schweiz
}

\section{Schlüsselwörter \\ Chronische Erschöpfung · Behandlung}

\section{Zusammenfassung}

Die Behandlung chronischer Erschöpfungssyndrome wurde und wird kontrovers diskutiert, was trotz aller Eindeutigkeit der empirischen Faktenlage auf die divergierenden Annahmen zu den Ursachen der chronischen Erschöpfung und auf die Zielsetzungen der abgeleiteten therapeutischen Vorgehensweisen zurückzuführen ist. Interessanterweise lassen sich die wirksamen Behandlungen nicht durch die von ihnen postulierten Mechanismen erklären. Unter Berücksichtigung früherer und historischer Behandlungsansätze chronischer Erschöpfungssyndrome soll das Rational aktueller Vorgehensweisen kritisch hinterfragt und die Notwendigkeit weiterer Forschung zu den Veränderungsmechanismen hervorgehoben werden.

\section{Behandlung ohne Ursache?}

In einem Kommentar zu den Ergebnissen einer Internettherapie bei chronisch erschöpften Jugendlichen [Nijhof et al., 2012] wiesen White und Chalder [2012] darauf hin, dass eine wirksame Therapie auch ohne bekannte Ursache der damit behandelten Erkrankung oder Störung möglich ist. Diese Aussage zielt auf die Tatsache, dass die (somatische) Ursache chronischer Erschöpfungssyndrome - nach Verlust einer sicher geglaubten viralen Genese [Alberts, 2011; Gaab, 2011] - nach wie vor als unbekannt gilt, wohl aber effektive Behandlungsmöglichkeiten vorliegen. Diese Aussage und deren Schlussfolgerung ist insofern interessant, als die aktu-

\author{
Keywords \\ Chronic fatigue $\cdot$ Treatment
}

\section{Summary}

Go West or Rest! The Treatment of Chronic Fatigue

Syndromes Down the Years

Due to opposing assumptions concerning the ultimate causes of fatigue and the interventional goals and despite clear-cut empirical results, the treatment of chronic fatigue syndromes has been and still is subject to controversy. Even though efficacious interventions are now established, their effects cannot be fully explained by their proposed mechanisms. With regard to historical treatment concepts of chronic fatigue syndromes, the rationale of current approaches is critically discussed, and the necessity of further research on the mechanisms of amendment is underlined. ell diskutierten und evaluierten Behandlungsansätze bei funktionalen chronischen Erschöpfungszuständen [zur Übersicht: Malouff et al., 2008; Martin und Gaab, 2011] tatsächlich auf ätiologischen Modellen basieren und sich auch die aktuell diskutierten therapeutischen Vorgehensweisen aufgrund divergierender Annahmen zu Ursache, Aufrechterhaltung und Vorgehensweise stark unterscheiden. Am Beispiel einer aktuellen und viel diskutierten Studie [White et al., 2011b] sollen im Folgenden die grundlegenden Annahmen sowie die daraus abgeleiteten Interventionsvorgehen beschrieben und mit Blick auf ihre historischen Vorläufer sowie auf die noch ausstehenden Forschungsfragen kritisch diskutiert werden.

\section{KARGER \\ Fax +497614520714 \\ Information@Karger.com}

www.karger.com (c) 2014 S. Karger GmbH, Freiburg

$1016-6262 / 14 / 0242-0108 \$ 39.50 / 0$

Accessible online at:

www.karger.com/ver 


\section{Chronische Erschöpfung - eine wenig greifbare Störung}

Betroffene wie auch Behandelnde von chronischer Erschöpfung stehen vor der schweren Aufgabe, ein unspezifisches und nicht spezifizierbares Symptom, das sich weder durch aktuelle oder starke Belastungen noch durch eine eindeutig nachweisbare somatische Ursache oder psychische Störung erklären lässt, zu verstehen und aus diesem Verständnis eine Behandlung abzuleiten. Diese Schwierigkeiten lassen sich leicht auch in der Wissenschaft und deren Rezeption erkennen: So gibt es in der neueren Erschöpfungsforschung (Eine erste Erwähnung chronischer Erschöpfung als «Febricula» wird auf die Mitte des 18. Jahrhunderts datiert. Ab 1869 wurde daraus (bis heute in der International Classification of Diseases 10 (ICD-10) als F48.8) die Neurasthenie, 1955 kam es zur lokalen Epidemie der Myalgischen Enzephalomyelitis (bis heute im ICD-10 als G93.3) [The Medical Staff of the Royal Free Hospital, 1957]. Die aktuelle Neuzeit der Erschöpfungsforschung begann mit den 1988-Kriterien des Center for Disease Control für das Chronic Fatigue Syndrome (CFS).) insgesamt acht verschiedene Definitionsansätze, die nicht nur unterschiedliche Spezifität und Sensibilität und damit entsprechend unterschiedliche Prävalenzschätzungen zur Folge haben, sondern auch den Vergleich zwischen Studien und über die Zeit maßgeblich erschweren [Johnston et al., 2013; Hamilton et al., 2005; White, 2007]. Aufgrund der großen Überlappung der somatischen Symptome ist eine eindeutige symptomatische Unterscheidung von depressiven Störungen nur nach ausführlicher differenzialdiagnostischer Abklärung [Dörr und Nater, 2013] bzw. vor allem anhand der subjektiven Krankheitsannahmen möglich [Moss-Morris und Petrie, 2001], sodass auch hier bei fehlender eindeutiger somatischer Ursache und hoher psychischer Komorbidität - eine eindeutige Positionierung als eigenständige nosologische Entität umstritten ist [Wessely und White, 2004]. So ist es wenig verwunderlich, dass sich die Annahmen und Positionen zu den Ursachen und der Behandlung von Betroffenen, in den Medien und aus wissenschaftlicher Sicht, deutlich unterscheiden [Hossenbaccus und White, 2013; Knudsen et al., 2011]. Die Problematik einer unspezifischen Symptomatik mit unklarer Ursache zeigt sich auch in der Kernzelle der Behandlung, der Konsultation zwischen Behandler und Patient: Patienten mit unspezifischen und nicht lokalisierbaren Symptomen werden als schwierig wahrgenommen [Raine et al., 2004], erleben die Erklärungen des behandelnden Arztes als Ablehnung eigener Erklärungen [Salmon et al., 1999] und erhalten zudem medizinische Behandlungen, ohne diese explizit verlangt zu haben [Ring et al., 2004].

\section{Neuroendokrine Dysfunktionen: Zentrale Henne oder peripheres Ei?}

Auch wenn wiederholt der Nachweis einer objektivierbaren monokausalen Ursache chronischer Erschöpfungssyndrome gefunden und anschließend wieder dementiert oder widerlegt wurde [Gaab, 2011], ist die Notwendigkeit und Sinnhaftigkeit der Frage nach einem physiologischen Substrat der erlebten Beschwerden nicht von der Hand zu weisen - der Umkehrschluss wäre sonst das oft befürchtete und falsche «Das ist nur eingebildet» sowie das nachvollziehbare «Ich bin doch nicht verrückt!».

Die Hypothalamus-Hypophysen-Nebennierenrinden-Achse (HHNA) wird aufgrund ihrer Funktion als Koordinatorin von psychobiologischen Bewältigungsprozessen als zentrales Bindeglied eines multifaktoriellen und biopsychosozialen Erklärungsmodells chronischer Erschöpfungssyndrome angesehen [Gaab, 2004]. Die Ergebnisse bisheriger Untersuchungen zeigen ein differenziertes Bild einer hypofunktionalen HHNA im Sinne eines moderaten Hypocortisolimus, einer verminderten Variabilität des Cortisolspiegels über den Tagesverlauf, einer erhöhten negativen Feedbacksensitivität und einer reduzierte HHNA-Reaktivität auf Belastungen [Papadopoulos und Cleare, 2011].

Noch nicht abschließend geklärt sind die Fragen nach der genauen Lokalisation der neuroendokrinen Dysfunktionen und der zeitliche Genese und deren klinische Bedeutung. Auch wenn wiederholt eine verringerte Cortisolsekretion beobachtet wurde [z.B. Nater et al., 2008], deuten Ergebnisse von unstimulierten [Di Giorgio et al., 2005] und reaktiven Messungen zentraler HHNA-Parameter [Gaab et al., 2002b] darauf hin, dass der Ursprung einer hypofunktionalen HHNA in einer reduzierten zentralen Aktivität und Reaktivität liegt, die möglicherweise durch ein erhöhte negative Feedbackregulation bedingt wird [Gaab et al., 2002a]. Interessanterweise konnte insgesamt eine physiologische Kompensation verschiedener peripherer Zielorgane und -zellen auf eine reduzierte Stimulation durch HHNA-Parameter beobachtet werden, wie beispielsweise eine Sensibilisierung auf Höhe der Nebennierenrinde und in immunologischen Zellpopulationen [Gaab et al., 2003a, 2003b].

In Bezug auf die Entwicklung dieser neuroendokrinen Dysfunktionen stellt sich die wichtige Frage, ob sie primärer oder sekundärer Natur sind. Gegen eine genuin primäre Rolle spricht, dass einerseits HHNA-Parameter in longitudinalen Studien keine Prädiktoren von chronischer Erschöpfung sind [Candy et al., 2003; Rubin et al., 2005] und dass andererseits das Ausmaß der neuroendokrinen Hypofunktion mit der Dauer der Störung positiv assoziiert ist [Gaab et al., 2004]. Auch wenn momentan noch ungeklärt ist, wie im Verlauf einer chronischen Erschöpfung eine hypofunktionale HHNA entsteht - Veränderungen des Aktivitäts- und Schlafverhaltens sowie psychische Belastungen und Störungen sind hier die aussichtsreichsten Erklärungsansätze [Tak et al., 2011] müssen auch frühe traumatische Erfahrungen als prädisponierende Risikofaktoren betrachtet werden [Heim et al., 2009]. 
Die empirische Absicherung der klinischen Bedeutsamkeit dieser neuroendokrinen Veränderungen steckt noch in den wissenschaftlichen Anfängen, wobei pharmakologische Behandlungen mit Glucocorticoiden keine symptomatischen Effekte aufweisen [Whiting et al., 2001]. Umgekehrt führen aber symptomatische Veränderungen im Kontext psychotherapeutischer Behandlungen $\mathrm{zu}$ moderaten Normalisierungen der HHNA-Aktivität [Roberts et al., 2009; Nijhof et al., 2014] und darüber hinaus ist eine hypofunktionale HHNA ein Prädiktor für einen geringeren Behandlungseffekt [Jason et al., 2007b; Roberts et al., 2010].

\section{Von der Ätiologie zur Intervention: Raste oder roste?}

Die ätiologische Diskussion dreht sich nicht nur um die Frage, ob den Symptomen ein physiologisches Substrat zugrunde liegt, sondern vielmehr darum, ob sie primär sind oder sekundär im Verlauf der Störung entstehen [White, 2000]. Diese Frage hat maßgeblichen Einfluss auf die therapeutische Kontroverse: Muss man sich und sein Leben den Symptomen anpassen oder ist das chronische Erschöpfungssyndrom reversibel? Diese Kontroverse gewinnt ihre Brisanz nicht nur aus der Erkenntnis, dass die Prognose bei Nicht-Behandlung düster ist [Cairns und Hotopf, 2005], sondern vor allem aus den sich widersprechenden Zielen der diskutierten Behandlungsansätze.

Auf der einen Seite steht das Angst-Vermeidungsmodell: Angst vor Aktivitäten und Belastungen führt zu Aktivitätsreduktion und -vermeidung; diese bedingen eine sekundäre physiologische Dekonditionierung, die wiederum zu körperlichen Symptomen führen und damit einer weiteren Aktivitätsreduktion Vorschub leisten kann. Vereinfacht ausgedrückt kommt es hier aufgrund der körperlichen Beschwerden zu einem Angst-Vermeidungskreislauf mit einer daraus resultierenden Hilf- und schlussendlich Hoffnungslosigkeit, wobei dieser Prozess von einer zunehmenden physiologischen Dekonditionierung begleitet wird. Daraus abgeleitet ist der Behandlungsansatz der Aktivitätssteigerung, entweder direkt durch ein mehr oder minder simples Aktivitätssteigerungsprogramm (allmählicher Aktivitätsaufbau [Van Cauwenbergh et al., 2012]) oder indirekt durch die Modifikation von aktivitätsbezogenen Kognitionen inklusive Verhaltensexperimenten (kognitive Verhaltenstherapie [zur Übersicht: Gaab und Ehlert, 2005]).

Diesem Verständnis und Vorgehen konträr ist das Energy Envelope-Modell (Energie-Umschlag ist hier eine schlechte Übersetzung; gemeint ist die begrenzte/umschriebene Menge an verfügbarer Energie/Leistungsfähigkeit) und das daraus abgeleitete Vorgehen: Die Leistungsfähigkeit ist aufgrund einer unbekannten somatischen Dysfunktion beschränkt, und wenn sie überschritten wird, entsteht Erschöpfung. Eine Kontrolle der Erschöpfung ist nur durch Kontrolle und Reduktion des Leistungsverbrauchs möglich. Das Ausmaß die- ser Leistungskontrolle/-reduktion variiert: Das Energy Envelope-Modell geht von einem Nullsummenspiel aus; das $\mathrm{Pa}$ cing-Modell ist sehr ähnlich und schlägt eine größtmögliche Aktivität im Rahmen der durch die Beschwerden auferlegten Grenzen vor, und das adaptive Pacing begrenzt das Ausmaß der Aktivität auf 70\% der Leistungsfähigkeit [zur Übersicht: Jason et al., 2013].

\section{PACE und where to PACE from here?}

Von den beiden beschriebenen (Erklärungs- und) Behandlungsansätzen chronischer Erschöpfungssyndrome wurden bislang vor allem kognitiv-verhaltenstherapeutische und genuin aktivitätssteigernde Intervention wiederholt evaluiert, wobei eine moderate Wirksamkeit beobachtet wurde [zur Übersicht: Edmonds et al., 2004; Price et al., 2008]. Die Validität dieser wissenschaftlichen Ergebnisse sowie die Anwendbarkeit und Sicherheit aktivitätssteigernder Interventionen wurde (und wird) aber von Selbsthilfegruppen [Kindlon, 2011] und vereinzelt auch von wissenschaftlicher Seite angezweifelt [Song und Jason, 2005]. In einer großen randomisiert-kontrollierten Multicenter-Studie an 640 Patientinnen und Patienten mit chronischem Erschöpfungssyndrom und unter Einbeziehung von Selbsthilfegruppen wurde erstmalig ein direkter Vergleich der beiden Interventionsansätze (d.h. Aktivitätsanpassung vs. Aktivitätssteigerung) durchgeführt ( $p$ acing, graded activity, and cognitive behaviour therapy: a randomised evaluation (PACE)) [White et al., 2011b]. Zusammengefasst zeigten die Ergebnisse, dass ein allmählicher Aktivitätsaufbau und eine kognitiv-verhaltenstherapeutische Behandlung im langfristigen Verlauf über 52 Wochen einer Aktivitätsanpassung/Pacing sowie einer ausschließlich medizinischen Betreuung in Bezug auf die primären Endpunkte (Erschöpfung und körperliche Leistungsfähigkeit) überlegen waren und dass sich die vier Interventionen in Anzahl und Ausmaß der unerwünschten Ereignisse nicht unterschieden. Weitere Studien konnten zeigen, dass sich die Interventionen in ihrer Kosteneffizienz (kognitive Verhaltenstherapie > allmählicher Aktivitätsaufbau $>$ Aktivitätsanpassung $=$ medizinische Betreuung) $[$ McCrone et al., 2012], in der Reduktion von Muskel- und Gelenkschmerzen [Bourke et al., 2013] sowie in der Remissionsrate (kognitive Verhaltenstherapie $=$ allmählicher Aktivitätsaufbau $>$ Aktivitätsanpassung $=$ medizinische Betreuung) [White et al., 2013] signifikant unterscheiden und dass sich vergleichbare Effekte auch in der (britischen) Versorgungsrealität beobachten lassen [Crawley et al., 2013].

Die Reaktionen von Betroffenen und Selbsthilfeorganisationen auf diese qualitativ hochstehende und in Bezug auf Größe und Durchführung einzigartige Studie war, wie (leider) zu erwarten war, negativ bzw. so negativ, dass sich die Editoren von The Lancet - bei aller berechtigten Kritik aufgrund der Veränderung des Studienprotokolls während der Studie und der Auswahl und Definition der primary und secondary 
outcomes [White et al., 2011a] - zu einem mäßigenden Aufruf veranlasst sahen [The Lancet, 2011]. Ungeachtet dieser Kritik und der wichtigen Klärung zentraler Fragen in der Behandlung chronischer Erschöpfung («Was ist effektiv und sicher?») wirft die PACE-Studie die Frage nach den Veränderungsprozessen auf, oder wie Bleijenberg und Knoop [2011] fragen: «Where do we PACE from here?»

Die beschriebenen ätiologischen Annahmen und die daraus abgeleiteten Vorgehensweisen lassen vermuten, dass die beobachteten Interventionseffekte durch eine Aktivitätssteigerung bedingt werden. Dies scheint aber weder beim allmählichen Aktivitätsaufbau [Moss-Morris et al., 2005] noch bei der kognitiven Verhaltenstherapie [Wiborg et al., 2010] der Fall zu sein. Vielmehr konnten für beide Interventionen Veränderungen der Symptomfokussierung als Mediatoren für die beobachteten Symptomreduktionen nachgewiesen werden [Moss-Morris et al., 2005; Wiborg et al., 2011]. Darüber hinaus konnte in einer Reanalyse einer wirkungsäquivalenten Vergleichsstudie zwischen kognitiver Verhaltenstherapie und personenzentrierter Psychotherapie gezeigt werden [Ridsdale et al., 2001], dass die emotionale Prozessierung, verstanden als Ausdruck, Anerkennung und Akzeptanz von emotionalen Belastungen, der wichtigste Prädiktor für eine Reduktion der Erschöpfung war [Godfrey et al., 2007]. Die Bedeutung der Emotionsregulation im sozialen Kontext zeigt sich auch in einer prospektiven Studie von Prins et al. [2004], bei der negative soziale Interaktionen einerseits die Erschöpfung im Verlauf vorhersagten und andererseits eine kognitiv-verhaltenstherapeutische Behandlung zu einer Reduktion der negativen Interaktionen führte.

Zudem fällt bei genauerer Betrachtung der PACE-Ergebnisse [White et al., 2011b] auf, dass über alle Interventionen die stärksten positiven Veränderungen zwischen der Baselineund der 12-Wochenmessung auftraten (durchschnittliche Effektstärke über alle Gruppen zwischen 0 und 12 Wochen: $\mathrm{d}=$ $0,85$, Spanne $0,80<\mathrm{d}<0,96)$, wobei dann im weiteren Verlauf bis zur 52. Woche in der Aktivitätsanpassung/Pacing sowie der ausschließlich medizinischen Betreuung eine sehr geringe (durchschnittliche Effektstärke über alle Gruppen zwischen 12 und 52 Wochen: $0,07<\mathrm{d}<0,16)$ und unter den aktivitätssteigernden Bedingungen noch eine mittlere Effektstärkenzunahme $(0,30<\mathrm{d}<0,45)$ beobachtet wurde. Vergleichbare Symptomreduktionen konnten auch in einer weiteren Vergleichsstudie (allmählicher Aktivitätsaufbau vs. personenzentrierte Beratung vs. medizinische Betreuung) bei chronisch erschöpften Patientinnen und Patienten in der medizinischen Erstversorgung beobachtet werden [Ridsdale et al., 2012]. Diese bedeutsamen (und methodenunspezifischen!) Effekte können nur schlecht durch den natürlichen Verlauf [Cairns und Hotopf, 2005] erklärt werden und ähneln eher einem Sudden Gain-Effekt, der wiederholt bei Angst- und depressiven Störungen beobachtet wurde [Aderka et al., 2012]. Auch wenn der Sudden Gain-Effekt nicht nur auf die Erwartungen von PatientInnen (und BehandlerInnen!) reduziert werden kann, spielen diese in der Anfangsphase der Therapie sicher- lich eine Rolle. In Anbetracht der deutlich niedrigeren Erwartungseffekte von chronisch erschöpften PatientInnen bei psychologisch-psychiatrischen Interventionen [Cho et al., 2015] liegt hier sicherlich noch eine Optimierungsmöglichkeit. Eine gemeinsame Festlegung der Behandlungsziele liefert dazu einen wichtigen Beitrag, der dann zu mehr Zustimmung zum Vorgehen, weniger Widerstand und Vermeidung und weniger therapieinkongruenter Symptombehandlung führt [Daniels und Wearden, 2011].

\section{Rest Cure und West Cure}

Bei aller Kontroverse und Begeisterung um die PACEStudie muss man sich fragen, ob die zur Diskussion stehenden Interventionen ausschließlich ein Resultat des in letzter Zeit stark angewachsenen Wissensstands in Sachen chronischer Erschöpfung sind. Zur Beantwortung dieser Frage sollen zwei historische Behandlungsansätze chronischer Erschöpfungssyndrome betrachtet werden.

In einem Londoner Hospital brach zwischen Juli und November 1955 eine obscure illness aus, die durch Kopfschmerzen, starke Malaise und weitere grippeähnliche Symptome gekennzeichnet war und nach ihrer vermuteten Prognose und Genese benigne myalgische Enzephalomyelitis (ME) genannt wurde. Insgesamt 292 Angehörige der Ärzteschaft und des Krankenhauspersonals waren betroffen (und interessanterweise nur 12 PatientInnen). Eine eindeutige somatische Ursache konnte nicht nachgewiesen werden, sodass diese lokale Epidemie im Nachhinein als eine der historischen Manifestationen von medizinisch unerklärten chronischen Erschöpfungszuständen betrachtet wird [Wessely, 1991]. Interessant ist aber, dass schon damals ein aus heutiger Sicht sinnvolles Behandlungsvorgehen empfohlen wurde: «Ruhe war der Rettungsanker in der akuten Phase. (...) Eine zuversichtliche und beruhigende Haltung der behandelnden ÄrztInnen war von großem Wert. (...) In der Rekonvaleszenz waren Beschäftigungs- und Spieltherapien sehr hilfreich» [The Medical Staff of the Royal Free Hospital, 1957, S. 899-900; Übersetzung des Autors]. Der Genesungsverlauf wurde durch großzügige Krankschreibungen unterstützt, der Wiedereinstieg durch eine Halbierung des Arbeitspensums begünstigt. Passive physiotherapeutische Maßnahmen wurden schon in der akuten Phase begonnen und im weiteren Verlauf der Genesung allmählich zum aktiven Aktivitätsaufbau ausgebaut, der aber dem Trainingszustand angepasst wurde, «to avoid musle spasms and cramps» [The Medical Staff of the Royal Free Hospital, 1957, S. 900]. Kam es aber dennoch zu solchen Nebenerscheinungen, wurden die bewegungstherapeutischen Maßnahmen dann in warmem Wasser durchgeführt.

Die Behandlung der Neurasthenie in ihrer Blütezeit Ende des 19. Jahrhunderts, also zwei Generationen vor der MEEpidemie in London, ist dagegen weniger patientenzentriert (patient-centered), sondern erscheint heute als Abbild eines 
misogynen Verständnisses chronischer Erschöpfung. Frauen wurden demnach aufgrund ihrer Disposition und ihrer vielfältigen Aufgaben als grundsätzlich schwächer und durch das Leben als Frau geschwächt angesehen, und entsprechend unterschied sich auch das therapeutische Vorgehen bei Männern und Frauen. Wurde chronisch erschöpften Frauen in der Regel eine andauernde und oftmals vollständige Bettruhe (rest cure) und damit eine konzentrierte Dosis von dem verabreicht, was, wie und wo man(n) sie gerne sah (ruhig, zu Hause und ohne intellektuelle Tätigkeiten), kamen die männlichen Betroffenen in den Genuss einer Aktivitätssteigerung an der frischen Luft: Aufenthalte in der Natur, inklusive Kraftübungen, Jagdausflüge, Camping und Reitausflüge in den damals noch wilden Westen Nordamerikas [Stiles, 2012]. Diese west cure war beliebt und bereitete unter anderem den Weg zur Entwicklung und Archetypisierung des American Wild West, inklusive Wildwestliteratur und -malerei, sowie zur Schaffung von Nationalparks [Historical Collections at the Claude Moore Health Sciences Library, 2007] und kann auch als Vorläufer heutiger Outdoor/Survival-Programme zur BurnoutPrävention und Rehabilitation angesehen werden.

Auch wenn die Effektivität der beschriebenen Behandlungsansätze nicht nach heutigen methodischen Standards erfasst und entsprechend nicht abschließend eruierbar ist, können die prominente Platzierung sowie die große Beliebtheit und Verbreitung dieser Interventionen als Beleg für eine gewisse Wirksamkeit angesehen werden.

\section{Behandlung ohne Ursache? Ein Fazit}

Es kann kein Zufall sein, dass sich die aktuellen, damaligen und historischen Behandlungsverfahren in der Umsetzung sehr ähneln. Der Grund dafür kann nicht in den zugrunde liegenden ätiologischen Modellen liegen; diese sind weder übereinstimmend noch - jedenfalls mit Blick auf die beschriebenen historischen Beispiele - wissenschaftlich belegt. Vielmehr ist ein Grund für diese Ähnlichkeit der beiden hauptsächlichen Behandlungsverfahren - rest cure vs. west cure bzw. Aktivitätsreduktion vs. Aktivitätsaufbau darin zu suchen, dass sie sich weitgehend auf den gesunden Menschenverstand (common sense) stützen. Betrachtet man aber einerseits das wachsende biopsychosoziale Verständnis chronischer Erschöpfungssyndrome und andererseits die Tatsache, dass die Therapieeffekte nicht oder nicht ausschließlich durch das erklärt werden, was sie eigentlich postulieren, dann sind die aktuellen Interventionsstudien [exemplarisch: White et al., 2011b] nicht das Ende, sondern der Beginn weiterer Forschung. Ansonsten gilt weiterhin: Behandlung ohne Ursache.

\section{Disclosure Statement}

Der Autor erklärt hiermit, dass keinerlei Interessenskonflikte in Bezug auf dieses Manuskript bestehen.

\section{Literatur}

Aderka IM, Nickerson A, Bøe HJ, Hofmann S: Sudden gains during psychological treatments of anxiety and depression: a meta-analysis. J Consult Clin Psychol 2012;80:93-101.

Alberts B: Retraction. Science 2011;334:1636.

Bleijenberg G, Knoop H: Chronic fatigue syndrome: where to PACE from here? Lancet 2011;377:786788.

Bourke JH, Johnson AL, Sharpe M, Chalder T, White PD: Pain in chronic fatigue syndrome: response to rehabilitative treatments in the PACE trial. Psychol Med 2013;23:1-8.

Cairns R, Hotopf M: A systematic review describing the prognosis of chronic fatigue syndrome. Occup Med (Lond) 2005;55:20-31.

Candy B, Chalder T, Cleare AJ, Peakman A, Skowera A, Wessely S, Weinman J, Zuckerman M, Hotopf M: Predictors of fatigue following the onset of infectious mononucleosis. Psychol Med 2003;33:847-855

Cho HJ, Hotopf M, Wessely S: The placebo response in the treatment of chronic fatigue syndrome: a systematic review and meta-analysis. Psychosom Med 2005;67:301-313.

Crawley E, Collin SM, White PD, Rimes K, Sterne JA, May MT; CFS/ME National Outcomes Database: Treatment outcome in adults with chronic fatigue syndrome: a prospective study in England based on the CFS/ME National Outcomes Database. QJM 2013;106:555-565.
Daniels J, Wearden AJ: Socialization to the model: the active component in the therapeutic alliance? A preliminary study. Behav Cogn Psychother 2011; 39:221-227.

Di Giorgio A, Hudson M, Jerjes W, Cleare AJ: 24Hour pituitary and adrenal hormone profiles in chronic fatigue syndrome. Psychosom Med 2005; 67:433-440.

Dörr J, Nater U: Erschöpfungssyndrome - Eine Diskussion verschiedener Begriffe, Definitionsansätze und klassifikatorischer Konzepte. Psychother Psychosom Med Psychol 2013;63:69-76.

Edmonds M, McGuire H, Price J: Exercise therapy for chronic fatigue syndrome. Cochrane Database Syst Rev 2004;(3):CD003200.pub2.

Gaab J: Psychotherapie chronischer Erschöpfungszustände. Psychotherapeut 2004;49:431-445.

Gaab J: Suche nach der Ursache des chronischen Erschöpfungssyndroms. Psychotherapeut 2011;56: 211-215.

Gaab J, Ehlert U: Chronische Erschöpfung und Chronisches Erschöpfungssyndrom. Fortschritte der Psychotherapie. Göttingen, Hogrefe Verlag, 2005.

Gaab J, Hüster D, Peisen R, Engert V, Heitz V, Schad T, Schürmeyer TH, Ehlert U: Hypothalamic-pituitary-adrenal axis reactivity in chronic fatigue syndrome and health under psychological, physiological, and pharmacological stimulation. Psychosom Med 2002a;64:951-962.
Gaab J, Hüster D, Peisen R, Engert V, Schad T, Schürmeyer TH, Ehlert U: Low-dose dexamethasone suppression test in chronic fatigue syndrome and health. Psychosom Med 2002b;64:311-318.

Gaab J, Hüster D, Peisen R, Engert V, Heitz V, Schad T, Schürmeyer T, Ehlert U: Assessment of cortisol response with low-dose and high-dose ACTH in patients with chronic fatigue syndrome and healthy com parison subjects. Psychosomatics 2003a;44:113-119.

Gaab J, Rohleder N, Heitz V, Schad T, Engert V, Schürmeyer TH, Ehlert U: Enhanced glucocorticoid sensitivity in patients with chronic fatigue syndrome. Acta Neuropsychiatr 2003b;15:184-191.

Gaab J, Engert V, Heitz V, Schad T, Schürmeyer TH, Ehlert U: Associations between neuroendocrine responses to the insulin tolerance test and patient characteristics in chronic fatigue syndrome. J Psychosom Res 2004;56:419-424.

Godfrey E, Chalder T, Ridsdale L, Seed P, Ogden J: Investigating the active ingredients of cognitive behaviour therapy and counselling for patients with chronic fatigue in primary care: developing a new process measure to assess treatment fidelity and predict outcome. Br J Clin Psychol 2007;46:253-272.

Hamilton WT, Gallagher AM, Thomas JM, White PD: The prognosis of different fatigue diagnostic labels: a longitudinal survey. Fam Pract 2005;22:383-388.

-Heim C, Nater UM, Maloney E, Boneva R, Jones JF, Reeves WC: Childhood trauma and risk for chronic fatigue syndrome: association with neuroendocrine dysfunction. Arch Gen Psychiatry 2008;66:72-80. 
Historical Collections at the Claude Moore Health Sciences Library: Neurasthenia and the Culture of Nervous Exhaustion - The American West. 2007. http://exhibits.hsl.virginia.edu/nerves/west/.

Hossenbaccus Z, White PD: Views on the nature of chronic fatigue syndrome: content analysis. JRSM Short Rep 2013;4:4.

Jason LA, Torres-Harding S, Friedberg F, Corradi C, Njoku MG, Donalek J, Reynolds N, Brown M, Weitner BB, Rademaker A, Papernik M, et al.: Nonpharmacologic interventions for CFS: a randomized trial. J Clin Psychol Med Settings 2007a;14:275-296.

Jason LA, Torres-Harding S, Maher K, Reynolds N, Brown M, Sorenson M, Donalek J, Corradi K, Fletcher MA, Lu T: Baseline cortisol levels predict treatment outcomes in chronic fatigue syndrome non-pharmacologic clinical trial. J Chronic Fatigue Syndrome 2007b;14:39-59.

- Jason LA, Brown M, Brown A, Evans M, Flores S, Grant-Holler E, Sunnquist M: Energy conservation/envelope theory interventions to help patients with myalgic encephalomyelitis/chronic fatigue syndrome. Fatigue 2013;1:27-42.

Johnston S, Brenu EW, Staines DR, Marshall-Gradisnik S: The adoption of chronic fatigue syndrome/ myalgic encephalomyelitis case definitions to assess prevalence: a systematic review. Ann Epidemiol 2013;23:371-376.

Kindlon T: Reporting of harms associated with graded exercise therapy and cognitive behavioural therapy in myalgic encephalomyelitis/chronic fatigue syndrome. Bull IACFS/ME 2011;(2):59-111.

Knudsen AK, Omenås AN, Harvey SB, Løvvik CM, Lervik LV, Mykletun A: Chronic fatigue syndrome in the media: a content analysis of newspaper articles. JRSM Short Rep 2011;2:42.

Malouff JM, Thorsteinsson EB, Rooke SE, Bhullar N, Schutte NS: Efficacy of cognitive behavioral therapy for chronic fatigue syndrome: a meta-analysis. Clin Psychol Rev 2008;28:736-745.

Martin A, Gaab J: Evidenzbasierte Psychotherapie bei chronischer organisch unklarer Erschöpfung. Psychotherapeut 2011;56:231-238.

McCrone P, Sharpe M, Chalder T, Knapp M, Johnson AL, Goldsmith KA, White PD: Adaptive pacing, cognitive behaviour therapy, graded exercise, and specialist medical care for chronic fatigue syndrome: a cost-effectiveness analysis. PLoS One 2012;7:e40808.

Moss-Morris R, Petrie K: Discriminating between chronic fatigue syndrome and depression: a cognitive analysis. Psychol Med 2001;31:469-479.

Moss-Morris R, Sharon C, Tobin R, Baldi JC: A randomized controlled graded exercise trial for chronic fatigue syndrome: outcomes and mechanisms of change. J Health Psychol 2005;10:245-259.

- Nater UM, Maloney E, Boneva RS, Gurbaxani BM, Lin JM, Jones JF, Reeves WC, Heim C: Attenuated morning salivary cortisol concentrations in a population-based study of persons with chronic fatigue syndrome and well controls. J Clin Endocrinol Metab 2008;93:703-709.
Nijhof SL, Bleijenberg G, Uiterwaal CSPM, Kimpen JLL, van de Putte EM: Effectiveness of internetbased cognitive behavioural treatment for adolescents with chronic fatigue syndrome (FITNET): a randomised controlled trial. Lancet 2012;379:14121418.

Nijhof SL, Rutten JM, Uiterwaal CS, Bleijenberg G, Kimpen JL, Putte EM: The role of hypocortisolism in chronic fatigue syndrome. Psychoneuroendocrinology 2014;42:199-206.

Papadopoulos AS, Cleare AJ: Hypothalamic-pituitary-adrenal axis dysfunction in chronic fatigue syndrome. Nat Rev Endocrinol 2011;8:22-32.

Price JR, Mitchell E, Tidy E, Hunot V: Cognitive behaviour therapy for chronic fatigue syndrome in adults. Cochrane Database Syst Rev 2008;(3):CD001027.

Prins JB, Bos E, Huibers MJ, Servaes P, van der Werf SP, van der Meer JW, Bleijenberg G: Social support and the persistence of complaints in chronic fatigue syndrome. Psychother Psychosom 2004;73: 174-182.

Raine R, Carter S, Sensky T, Black N: General practitioners' perceptions of chronic fatigue syndrome and beliefs about its management, compared with irritable bowel syndrome: qualitative study. BMJ 2004:328:1354-1357.

Ridsdale L, Godfrey E, Chalder T, Seed P, King M, Wallace P, Wessely S; Fatigue Trialists' Group: Chronic fatigue in general practice: is counselling as good as cognitive behaviour therapy? A UK randomised trial. Br J Gen Pract 2001;51:19-24.

Ridsdale L, Hurley M, King M, McCrone P, Donaldson $\mathrm{N}$ : The effect of counselling, graded exercise and usual care for people with chronic fatigue in primary care: a randomized trial. Psychol Med 2012;42:2217-2224.

Ring A, Dowrick C, Humphris G, Salmon P: Do patients with unexplained physical symptoms pressurise general practitioners for somatic treatment? A qualitative study. BMJ 2004:328:1057.

Roberts AD, Papadopoulos AS, Wessely S, Chalder T, Cleare AJ: Salivary cortisol output before and after cognitive behavioural therapy for chronic fatigue syndrome. J Affect Disord 2009;115:280-286.

Roberts AD, Papadopoulos AS, Wessely S, Chalder T, Cleare AJ: Does hypocortisolism predict a poor response to cognitive behavioural therapy in chronic fatigue syndrome? Psychol Med 2010;40:515-522.

Rubin GJ, Hotopf M, Papadopoulos A, Cleare A: Salivary cortisol as a predictor of postoperative fatigue. Psychosom Med 2005;67:441-447.

Salmon P, Peters S, Stanley I: Patients' perceptions of medical explanations for somatisation disorders: qualitative analysis. BMJ 1999;318:372-376.

Song S, Jason LA: A population-based study of chronic fatigue syndrome (CFS) experienced in differing patient groups: an effort to replicate Vercoulen et al.'s model of CFS. J Ment Health 2005;14:277-289.

Stiles A: Go rest, young man. Monit Psychol 2012;43:32. www.apa.org/monitor/2012/01/go-rest.aspx.
Tak LM, Cleare AJ, Ormel J, Manoharan A, Kok IC, Wessely S, Rosmalen JG: Meta-analysis and metaregression of hypothalamic-pituitary-adrenal axis activity in functional somatic disorders. Biol Psychol 2011;87:183-194.

The Lancet: Patients' power and PACE. Lancet 2011 377:1808

The Medical Staff of the Royal Free Hospital: An outbreak of encephalomyelitis in the Royal Free Hospital Group, London, in 1955. BMJ 1957;19:895-904.

Van Cauwenbergh D, De Kooning M, Ickmans K, Nijs J: How to exercise people with chronic fatigue syndrome: evidence-based practice guidelines. Eur J Clin Invest 2012;42:1136-1144.

Wessely S: History of the postviral fatigue syndrome. Br Med Bull 1991;47:919-941.

Wessely S, White PD: There is only one functional somatic syndrome. Br J Psychiatry 2004;185:95-96.

White PD: The role of physical inactivity in the chronic fatigue syndrome. J Psychosom Res 2000;49:283-284.

White PD: How common is chronic fatigue syndrome; how long is a piece of string? Popul Health Metr 2007;5:6.

White PD, Chalder T: Chronic fatigue syndrome: treatment without a cause. Lancet 2012;379:1372-1373.

White PD, Goldsmith K, Johnson AL, Chalder T, Sharpe M: The PACE trial in chronic fatigue syndrome - Authors' Reply. Lancet 2011a;377:18341835.

White PD, Goldsmith KA, Johnson AL, Potts L, Walwyn R, DeCesare JC, Baber HL, Burgess M, Clark LV, Cox DL, Bavinton J, Angus BJ, Murphy G, Murphy M, O'Dowd H, Wilks D, McCrone P, Chalder T, Sharpe M; PACE Trial Management Group: Comparison of adaptive pacing therapy, cognitive behaviour therapy, graded exercise therapy, and specialist medical care for chronic fatigue syndrome (PACE): a randomised trial. Lancet 2011b;377:823-836.

-White PD, Goldsmith K, Johnson AL, Chalder T, Sharpe M: Recovery from chronic fatigue syndrome after treatments given in the PACE trial. Psychol Med 2013;43:2227-2235.

Whiting P, Bagnall AM, Sowden AJ, Cornell JE, Mulrow $\mathrm{CD}$, Ramirez $\mathrm{G}$ : Interventions for the treatment and management of chronic fatigue syndrome. J Am Med Assoc 2001;286:1360-1368.

Wiborg JF, Knoop H, Stulemeijer M, Prins JB, Bleijenberg G: How does cognitive behaviour therapy reduce fatigue in patients with chronic fatigue syndrome? The role of physical activity. Psychol Med 2010;40:1281-1287.

Wiborg JF, Knoop H, Prins JB, Bleijenberg G: Does a decrease in avoidance behavior and focusing on fatigue mediate the effect of cognitive behaviour therapy for chronic fatigue syndrome? J Psychosom Res 2011;70:306-310. 